\title{
Apatinib suppresses cell growth and metastasis and promotes antitumor activity of temozolomide in glioma
}

\author{
CHAO WANG $^{1}$, MAN JIANG ${ }^{2}$, HELEI HOU ${ }^{2}$, QIANG LIN $^{2}$, ZHIYONG YAN ${ }^{1}$ and XIAOCHUN ZHANG ${ }^{2}$ \\ Departments of ${ }^{1}$ Neurosurgery and ${ }^{2}$ Medical Oncology, The Affiliated Hospital of Qingdao University, \\ Qingdao, Shandong 266003, P.R. China
}

Received September 29, 2017; Accepted April 30, 2018

DOI: $10.3892 / \mathrm{ol} .2018 .9355$

\begin{abstract}
Apatinib, a small-molecule multi-targeted tyrosine kinase inhibitor, is widely used to treat various types of solid tumors. In the present study, it was investigated whether apatinib has therapeutic potential for glioma. Cell Counting Kit- 8 and colony formation assays were utilized to determine the cell viability of p53- and EGFR-mutated U251MG glioma cells, and wild-type U-87MG ATCC glioma cells. Furthermore, apoptosis, and the invasion and migration abilities of glioma cells were investigated by flow cytometry, invasion assays and wound-healing assays. The potential of the combination of apatinib with temozolomide (TMZ) for glioma therapy was also investigated. The results demonstrate that apatinib significantly inhibited cell proliferation and colony formation through promoting cell apoptosis in p53- and EGFR-mutated and wild-type glioma cells. Cell invasion and migration abilities were notably decreased following treatment with apatinib. Overall, the present study indicates a synergistic antitumor effect of apatinib and TMZ in glioma, and presents a basis for the use of apatinib in glioma treatment.
\end{abstract}

\section{Introduction}

Malignant glioma is the most common type of primary central nervous system tumor, accounting for $80 \%$ all brain and central nervous system tumors $(1,2)$. Although major advancements have been made in surgical treatment, postoperative chemotherapy, and radiation, the prognosis for patients with malignant gliomas remains poor, with an average survival of roughly 1 year after diagnosis (3). According to Chinese glioma genome atlas (CGGA) statistics, the overall survival (OS) time for glioblastoma [GBM, World Health Organization (WHO) grade IV] patients is only 14.4 months, while the

Correspondence to: Dr Xiaochun Zhang, Department of Medical Oncology, The Affiliated Hospital of Qingdao University, 16 Jiangsu Road, Shinan, Qingdao, Shandong 266003, P.R. China

E-mail: qddxzhangxc@163.com

Key words: glioma, apatinib, temozolomide 6-month, 1-, 3- and 5-year OS rates were 87, 61, 15 and 9\%, respectively (4). The aggressiveness of glioma arises from the proliferation and invasion capabilities of malignant glioma cells (5). Thus, controlling these characteristics is a potential therapeutic strategy for glioma treatment.

Temozolomide (TMZ) is a prodrug that forms O6-methylguanine (O6-MeG) adducts, causing cytotoxicity via mismatch with deoxythymidine residues, inducing apoptosis following processing by the mismatch repair system (6). TMZ is converted to its active compound 3-methyl-(triazen-1-yl) imidazole-4-carboxamide at a physiological $\mathrm{pH}$ (7). The activated form of $\mathrm{TMZ}$ readily crosses the blood-brain barrier, and is, therefore, commonly used for the treatment of primary and secondary intracranial neoplasms (7). Despite the clinical success of TMZ, a large number of patients respond poorly to this agent, at least in part due to intrinsic resistance of tumor cells to damage-induced cell death $(8,9)$. Therefore, the identification of a novel combined strategy for glioma treatment is required.

Gliomas are highly vascular and rich in vascular endothelial growth factor (VEGF), which promotes angiogenesis. Therefore, the anti-VEGF monoclonal antibody, bevacizumab, and anti-VEGF receptor (VEGFR) tyrosine kinase inhibitors (TKIs), including sunitinib and sorafenib, have been used for treatment of patients with glioma (10). Apatinib is an orally administered second-generation inhibitor of the phosphorylation of the tyrosine residues within the intracellular domain of VEGF receptor 2 (VEGFR2) (11). Apatinib prevents VEGF-induced phosphorylation of VEGFR2 and the subsequent downstream signaling responsible for the biological effects of $\operatorname{VEGF}(12,13)$. Preclinical studies have demonstrated that apatinib exhibits antitumor activity in patients with different types of malignant tumors $(14,15)$. In the present study, it was investigated whether apatinib has therapeutic potential in glioma. The inhibitory activity of apatinib on cell proliferation and invasion was investigated in vitro using the cell counting kit- 8 assay, colony formation assay, Matrigel-based invasion assay and wound-healing assay. The potential of the combination of apatinib with TMZ for glioma therapy was also studied.

\section{Materials and methods}

Cell culture and treatment. The U251MG and U-87MG ATCC cell lines were purchased from the American Type 
Culture Collection (ATCC) and cultured in Dulbecco's modified Eagle's medium (DMEM) with nutrient mixture: F12 (Gibco; Thermo Fisher Scientific, Inc., Waltham, MA, USA), supplemented with $10 \%$ fetal bovine serum (FBS; Gibco; Thermo Fisher Scientific, Inc.), without antibiotics. U-87MG ATCC cells were of CNS origin and are likely to be a bonafide human glioblastoma cell line considering their mRNA expression profile. Thus, U-87MG ATCC cells can be used in glioma research and are distinct from U87 Uppsala cells established in 1968 at the University of Uppsala (16). Cells were maintained at $37^{\circ} \mathrm{C}$ in a humidified atmosphere containing $5 \% \mathrm{CO}_{2}$. Apatinib (cat. no. S2221) and TMZ (cat. no. S1237) were purchased from Selleck Chemicals (Houston, Texas, USA) and used to treat U251MG and U-87MG ATCC cells. A stock solution was prepared in dimethyl sulfoxide at $10 \mathrm{mM}$ and was stored at $-20^{\circ} \mathrm{C}$.

Western blot analysis. Cells were washed twice with ice-cold PBS and lysed in ice-cold RIPA lysis buffer (Beyotime Institute of Biotechnology, Beijing, China) containing a protease/phosphatase inhibitor cocktail (Beyotime Institute of Biotechnology). The protein concentration was determined by BCA assay (Thermo Fisher Scientific, Inc.). Cell lysates were separated by 6 or $10 \%$ SDS-PAGE [6\% for VEGFR2, phospho- (p-)VEGFR2 and 10\% for Akt, p-Akt, extracellular signal-regulated kinase (Erk), p-Erk and GAPDH] and transferred onto polyvinylidene fluoride membranes (Merck KGaA, Darmstadt, Germany). After blocking with 5\% non-fat milk in Tris-buffered saline with Tween (TBST), the blots were incubated with the following primary antibodies: P-VEGFR2 (dilution, 1:600; cat. no. 2478), VEGFR2 (dilution, 1:1,000; cat. no. 9658), Akt (dilution, 1:1,000; cat. no. 4691), p-Akt (dilution, 1:1,000; cat. no. 4060), ERK (dilution, 1:1,000; cat. no. 4695), p-ERK (dilution, 1:1,000; cat. no. 4370) and GAPDH (dilution, 1:5,000, cat. no. 2118; all Cell Signaling Technology, Inc., Danvers MA, USA) at $4^{\circ} \mathrm{C}$ for overnight. Next, the membraned were incubated with a horseradish peroxidase-conjugated goat anti-mouse or rabbit secondary IgG antibody (cat. nos. ZDR5305 and ZDR5307; 1:10,000; OriGene Technologies, Inc., Beijing, China) at $37^{\circ} \mathrm{C}$ for $1 \mathrm{~h}$. Immunoreactive bands were visualized with a Immobilon ECL chemiluminescent reagent (EMD Millipore, Billerica, MA, USA). The band densities were quantified using ImagePro Plus (version 6.0; Media Cybernetics, Inc., Rockville, MD, USA).

Cell viability assay. U251MG and U-87MG ATCC cells were plated in 96-well plates at $1 \times 10^{3}$ cells/well), following treatment with $0,5,10$ or $20 \mu \mathrm{M}$ apatinib, and $20 \mu \mathrm{M} \mathrm{TMZ}$ as a previous study indicated (17) treatment for $48 \mathrm{~h}$, the viability was determined using Cell Counting Kit-8 (Dojindo Molecular Technologies, Inc., Kumamoto, Japan). The absorbance was measured at $450 \mathrm{~nm}$ and relative cell viability was calculated.

Colony formation assay. A $0.2-\mathrm{ml}$ layer of $0.5 \%$ low-melt agarose was diluted in complete growth medium (DMEM medium with 10\% FBS, Gibco; Thermo Fisher Scientific, Inc.) and plated in 6-well plates. A total of $1 \times 10^{3} \mathrm{U} 251 \mathrm{MG}$ or U-87MG ATCC cells were per well, with was DMEM medium containing 10\% FBS. Following $10 \mu \mathrm{M}$ apatinib and $20 \mu \mathrm{M}$ TMZ treatment for 12 days, the plate was fixed with
4\% paraformaldehyde and stained with $10 \%$ crystal violet (Beyotime Institute of Biotechnology) at room temperature (from 22 to $25^{\circ} \mathrm{C}$ ) for 15 mins. Visible plots were defined as a colony and the number of colonies in 3 separate wells were counted subsequent to scanned by light stereo microscope (magnification, $\mathrm{x} 4$ ).

Apoptosis detection assay. U251MG and U-87MG ATCC cells were plated in a 6 -well plate at $4 \times 10^{5}$ cells/well. Following $10 \mu \mathrm{M}$ apatinib and $20 \mu \mathrm{M} \mathrm{TMZ}$ treatment for $48 \mathrm{~h}$, the cells were harvested and stained using a Annexin V-FITC/PI Apoptosis Detection kit (Nanjing KeyGen Biotech Co., Ltd., Nanjing, China), according to the manufacturer's protocol. A flow cytometer (Acea Biosciences Inc., Zhejiang, China) was used to identify apoptotic cells and the apoptotic cells were analyzed using De Novo Software (version 1.2.4; Acea Biosciences Inc.).

Cell invasion assay. In vitro analysis of invasion was assessed using Matrigel (BD Biosciences, Frankling Lakes, NJ, USA) and $8-\mu \mathrm{m}$ Transwell inserts (BD Biosciences). The cell invasion assay was performed as previously described (18). Briefly, $50 \mu \mathrm{l}$ Matrigel (diluted 1:5 with serum-free DMEM) was plated onto the Transwell insert. Then a cell suspension of $5 \times 10^{3} \mathrm{U} 251 \mathrm{MG}$ or U-87MG ATCC cells was added. Treatments of $10 \mu \mathrm{M}$ apatinib and $20 \mu \mathrm{M}$ TMZ were made for $24 \mathrm{~h}$ at $37^{\circ} \mathrm{C}$. The invasive cells were fixed with $4 \%$ paraformaldehyde (Beyotime Institute of Biotechnology) and stained with $10 \%$ crystal violet (Beyotime Institute of Biotechnology) at room temperature (from 22 to $25^{\circ} \mathrm{C}$ ) for 15 mins. The invasive cells were photographed by light microscopy (DTX500; Nikon Corporation, Tokyo, Japan) with a magnification of $x 40$ and the cells in three random fields of view were counted.

Wound-healing assay. U251MG and U-87MG ATCC cells were grown to $90 \%$ confluency in 6 -well plates. A wound was made by dragging a plastic pipette tip across the cell surface. Phase contrast images of the wounds were taken at 0 and $48 \mathrm{~h}$ of treatment with $10 \mu \mathrm{M}$ apatinib and $20 \mu \mathrm{M}$ TMZ. Image $\mathrm{J}$ software (National Institutes of Health, Bethesda, MD, USA) was used to evaluate the migration rate of U251MG and U-87MG ATCC cells.

Statistical analysis. All data are presented as the mean \pm standard deviations. All experiments were performed $\geq 3$ times independently. Statistical analyses were performed using SPSS (version 19.0; IBM Corp., Armonk, NY, USA). Student $t$-tests were performed to compare differences between 2 groups. One-way analysis of variance followed by Tukey's test was used for multiple comparisons analysis. $\mathrm{P}<0.05$ was considered to indicate a statistically significant difference.

\section{Results}

Apatinib suppresses activation of VEGFR2 in glioma cells. To determine the potential role of apatinib in glioma-cell proliferation, p53- and EGFR-mutated (U251MG) and wild-type (U-87MG ATCC) cells were treated with 0, 5, 10 and $20 \mu \mathrm{M}$ apatinib. Western blotting was performed to detect p-VEGFR2 and VEGFR2 protein expression. Apatinib 

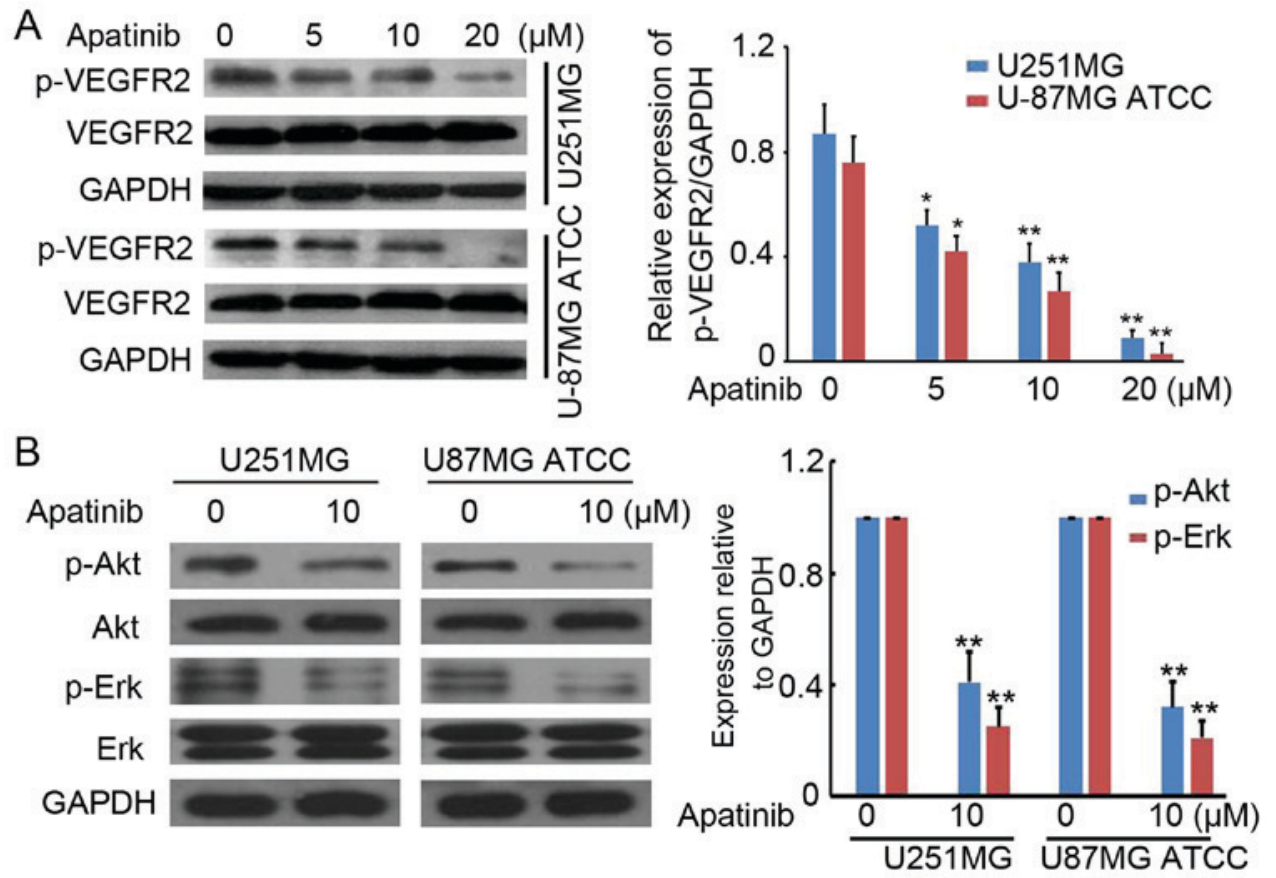

Figure 1. Apatinib suppresses VEGFR2 activation in glioma. (A) Western blotting analysis of p-VEGFR2 and VEGFR2 expression in U251MG and U-87MG ATCC cells following treatment with 0,5, 10 and $20 \mu \mathrm{M}$ apatinib for $48 \mathrm{~h}$. (B) Western blotting of p-Akt, Akt, p-ERK and ERK expression in U251MG and U-87MG ATCC cells following treatment with and without $10 \mu \mathrm{M}$ apatinib for $48 \mathrm{~h}$. ${ }^{*} \mathrm{P}<0.05,{ }^{* * *} \mathrm{P}<0.01$, compared with $0 \mu \mathrm{M}$ apatinib. P-, phosphorylated; VEGFR2, vascular endothelial growth factor receptor 2; ERK, extracellular signal-regulated kinase.

significantly inhibited $\mathrm{p}-\mathrm{VEGFR} 2$ protein expression in a concentration-dependent manner in U251MG and U-87MG ATCC cells (Fig. 1A). It was also suggested that the activation of downstream targets of VEGFR2 signaling pathway (Akt and ERK) was also inhibited by apatinib in glioma cells (Fig. 1B). These results confirm an inhibition role of apatinib in VEGFR2-activation in glioma cells.

Apatinib suppresses cell proliferation in glioma. To analyze the antitumor activity of apatinib in glioma, a CCK-8 assay was performed to determine the cell viability of U251MG and U-87MG ATCC cells following apatinib treatment at $0,5,10$ and $20 \mu \mathrm{M}$ for $48 \mathrm{~h}$. Apatinib efficiently impaired U251MG and U-87MG ATCC cell proliferation at concentrations of 10 and $20 \mu \mathrm{M}$, an effect which was concentration-dependent (Fig. $1 \mathrm{~A}$ and $\mathrm{B}$ ). The $\mathrm{IC}_{50}$ of apatinib was $\sim 13 \mu \mathrm{M}$ in U-87MG ATCC and U251MG cells. Therefore, a concentration of $10 \mu \mathrm{M}$ which was lower than $\mathrm{IC}_{50}$ of apatinib was used for colony formation and apoptosis detection assays. Inhibition of colony formation of U251MG and U-87MG ATCC cells was also identified following apatinib treatment $(\mathrm{P}<0.01$; Fig. $2 \mathrm{C}$ and $\mathrm{D})$. To determine the mechanism underlying apatinib-mediated inhibition of glioma-cell proliferation, flow cytometry was performed to investigate U251MG and U-87MG ATCC-cell apoptosis with or without apatinib. The results revealed an increased rate of apoptosis both in U251MG and U-87MG ATCC cells treated with apatinib compared with untreated cells $(\mathrm{P}<0.01$; Fig. $2 \mathrm{E}$ and $\mathrm{F})$. These results suggest that apatinib inhibits glioma-cell proliferation and colony formation through induction of apoptosis.

Apatinib suppresses cell invasion in glioma. Invasion and wound-healing assays were performed to investigate the invasion and migration abilities of U251MG and U-87MG ATCC cells upon apatinib treatment. As demonstrated in Fig. 3A and B, apatinib-treated U251MG and U-87MG ATCC cells were less invasive than untreated cells $(\mathrm{P}<0.01$; Fig. 3A and B). Furthermore, apatinib significantly inhibited the migratory ability of U251MG and U-87MG ATCC cells compared with control ( $\mathrm{P}<0.01$; Fig. $3 \mathrm{C}$ and $\mathrm{D})$. Collectively, these results demonstrate the inhibitory role of apatinib on cell invasion in glioma.

Apatinib promotes TMZ-mediated inhibition of proliferation in glioma. Based on the above results, it was investigated whether apatinib enhances TMZ-mediated inhibition of proliferation of glioma cells. The cell viability of U-87MG ATCC cells after treatment with apatinib with or without TMZ was determined by CCK- 8 assay. TMZ was used at a concentration of $20 \mu \mathrm{M}$ according to a previous study by Nitta et al (17). Apatinib or TMZ treatments alone efficiently inhibited proliferation of glioma cells, compared with untreated cells $(\mathrm{P}<0.01$; Fig. 4A). However, in combination, apatinib significantly enhanced TMZ-mediated inhibition of proliferation of U-87MG ATCC cells, compared with cells treated with TMZ alone $(\mathrm{P}<0.01$; Fig. $4 \mathrm{~A})$. Similar results were found in U251 cells (data not shown). A colony formation assay also demonstrated the enhancing role of apatinib on TMZ-mediated antitumor activity $(\mathrm{P}<0.01$, compared with cells treated with TMZ alone; Fig. 4B). An increased proportion of apoptotic U-87MG ATCC cells was also demonstrated in the group treated with apatinib and TMZ compared with U-87MG ATCC cells treated with TMZ alone $(\mathrm{P}<0.01$; Fig. 4C). Collectively, these results indicate that apatinib significantly improved the antitumor activity of TMZ in glioma. 

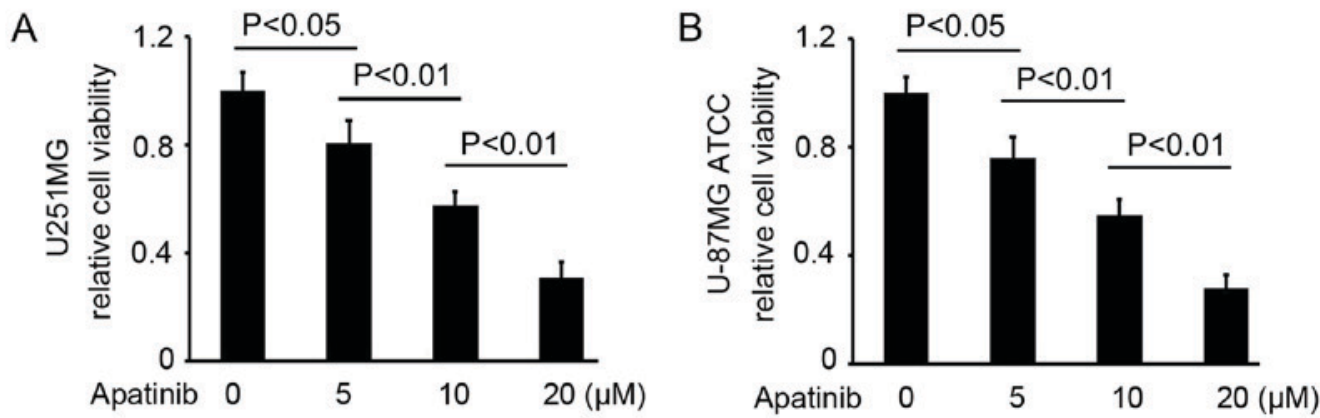

C
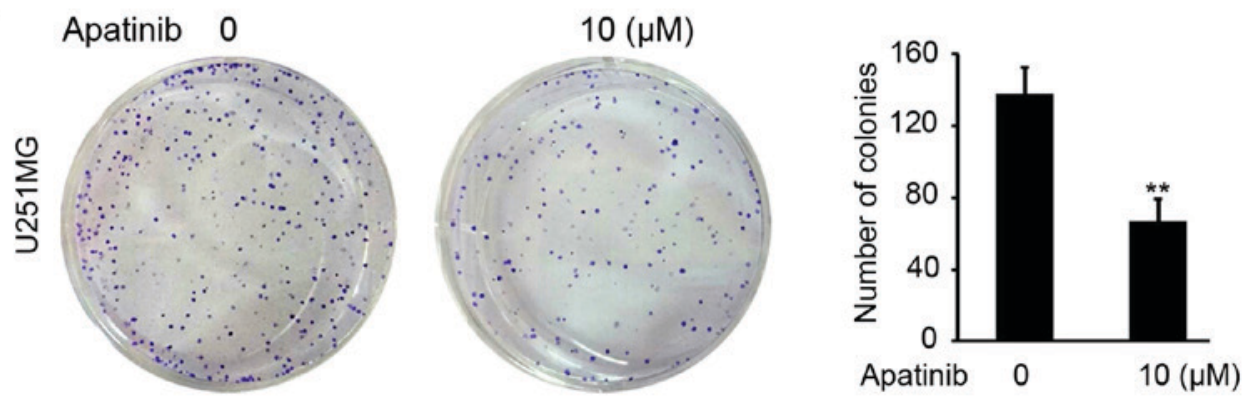

D Apatinib 0

$10(\mu \mathrm{M})$
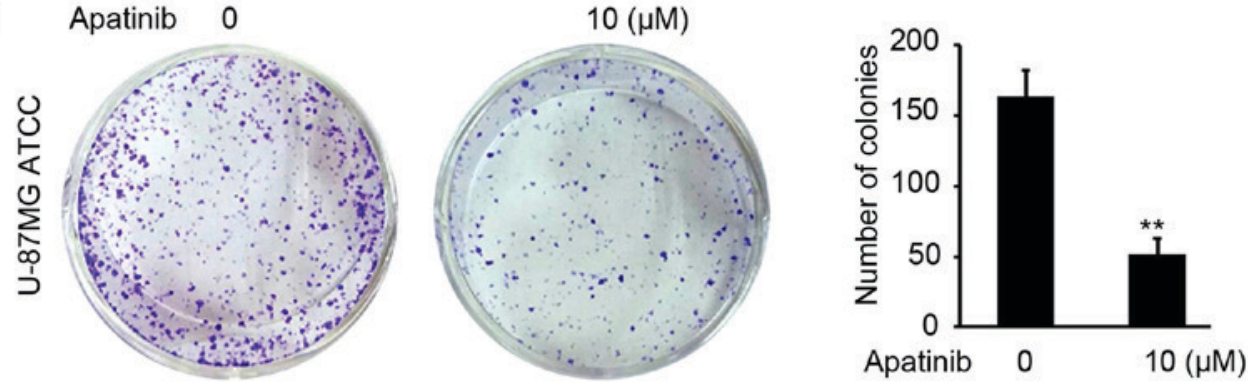

E
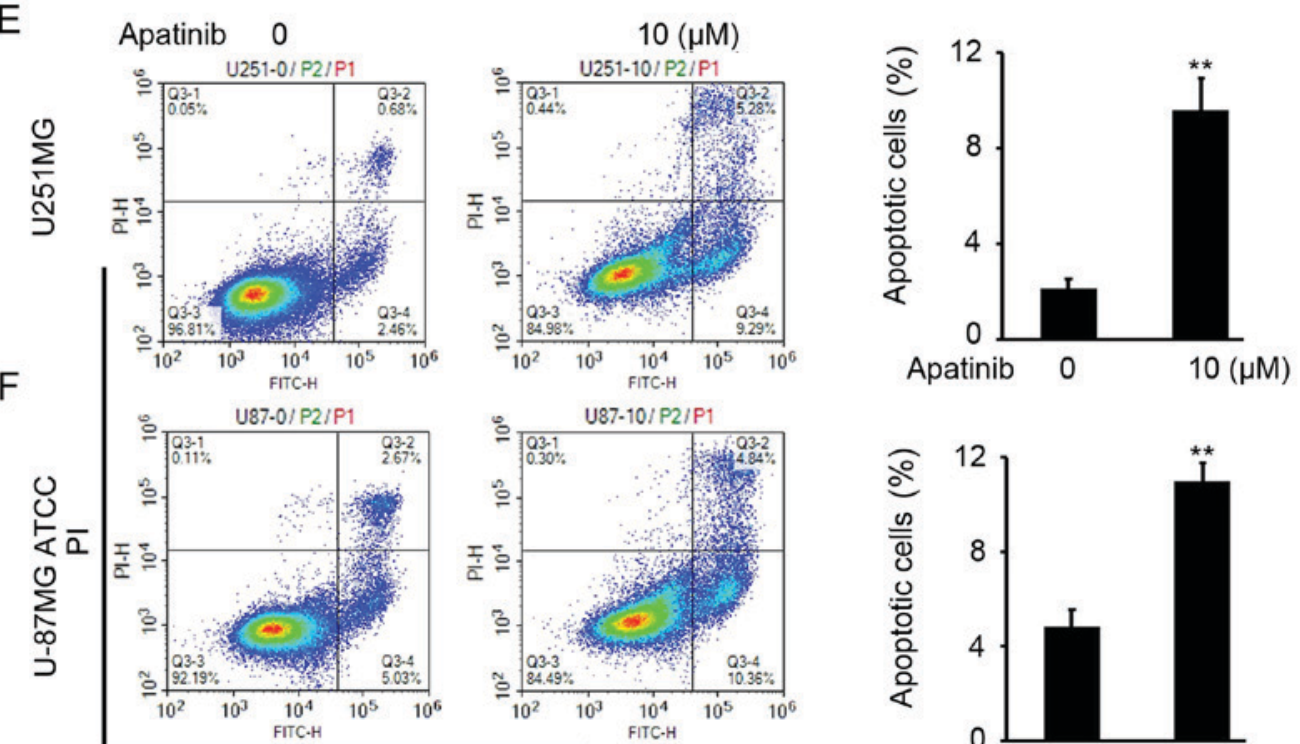

Annexin V-FITC

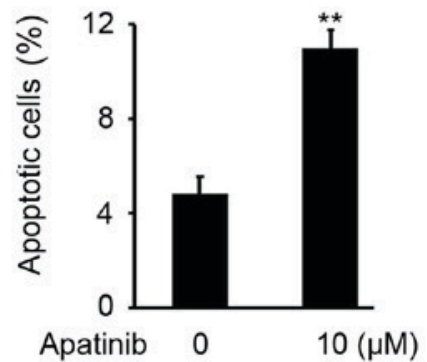

Figure 2. Apatinib suppresses proliferation of glioma cells. The cell viability of (A) U251MG cells, and (B) U-87MG ATCC cells was determined following treatment with $0,5,10$ and $20 \mu \mathrm{M}$ apatinib, relative to that of untreated cells. $\mathrm{n}=5$ for each group. Colony formation assays were performed in (C) U251MG cells, and (D) U-87MG ATCC cells following treatment with 0 and $10 \mu \mathrm{M}$ apatinib. The number of colonies was counted and analyzed. $\mathrm{n}=3$ for each group. ${ }^{* *} \mathrm{P}<0.01$ vs. $0 \mu \mathrm{M}$ apatinib. Analysis of cell apoptosis by Annexin-V/PI staining in (E) U251MG cells, and (F) U-87MG ATCC cells following treatment with 0 and $10 \mu \mathrm{M}$ apatinib. $\mathrm{n}=3$ for each group. ${ }^{* *} \mathrm{P}<0.01$ vs. $0 \mu \mathrm{M}$ apatinib. $\mathrm{PI}$, proprium iodide.

Apatinib improves TMZ-mediated inhibition of cell invasion in glioma. It was also investigated whether apatinib enhances TMZ-mediated inhibition invasion of glioma cells. As demonstrated Fig. 5A, a reduced number of invasive U-87MG ATCC cells were counted in the groups treated with apatinib and TMZ compared with control cells ( $\mathrm{P}<0.01$; Fig. $5 \mathrm{~A}$ and $\mathrm{B})$. 
A

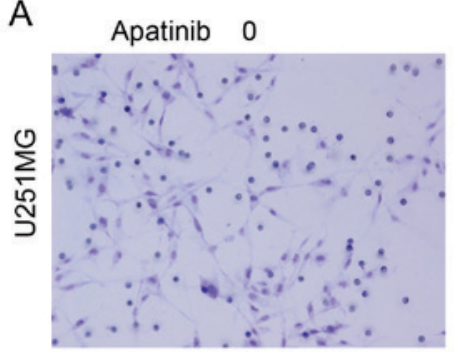

B

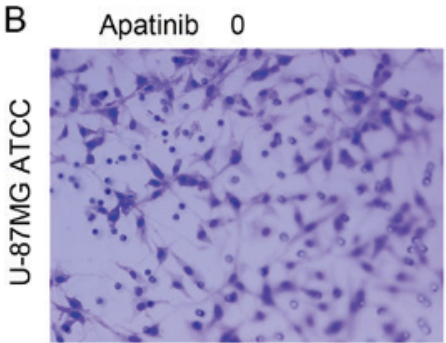

C
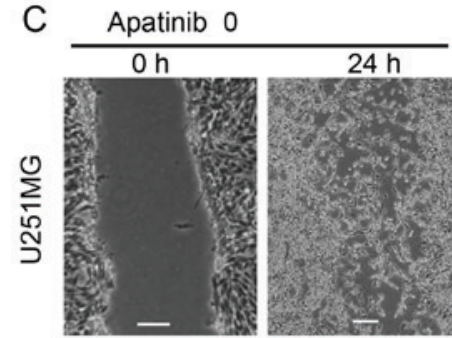

D Apatinib 0

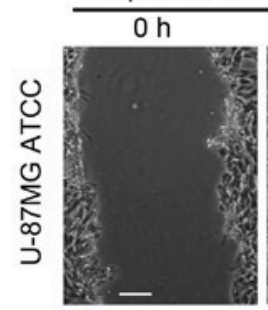

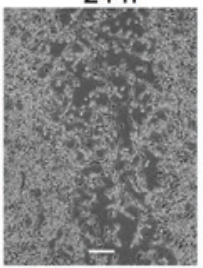

$24 \mathrm{~h}$

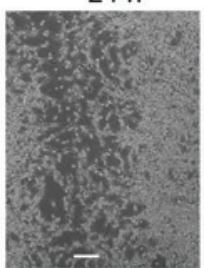

$10(\mu \mathrm{M})$

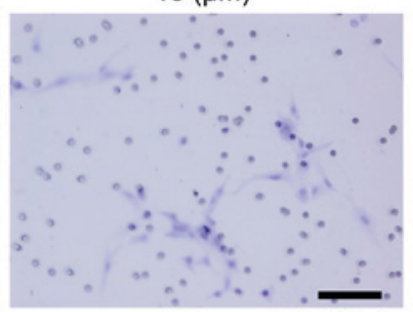

$10(\mu \mathrm{M})$
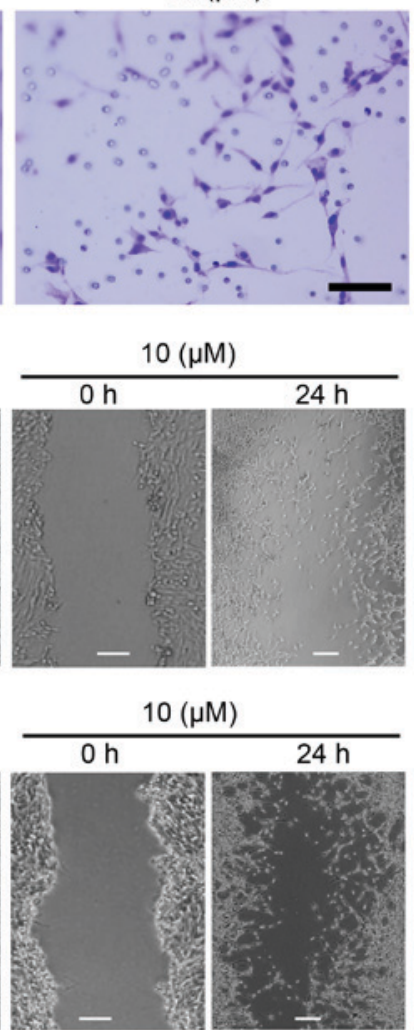
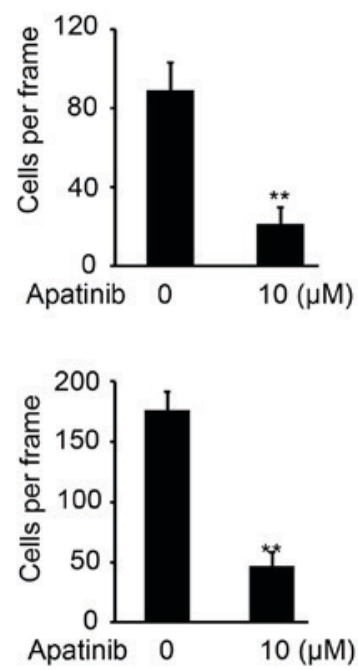

Apatinib $0 \quad 10(\mu \mathrm{M})$
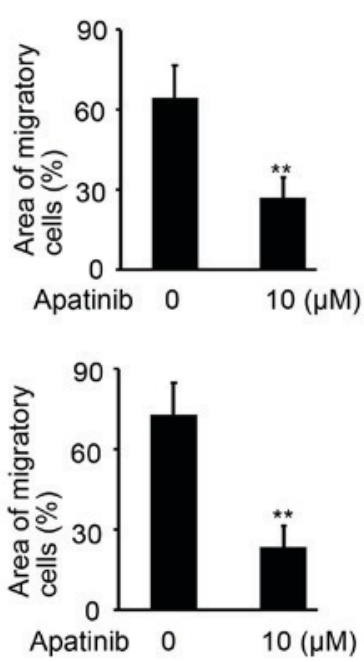

Figure 3. Apatinib suppresses glioma-cell migration. Invasion assays were performed to determine the invasive abilities of (A) U251MG cells, and (B) U-87MG ATCC cells following treatment with 0 and $10 \mu \mathrm{M}$ apatinib. The number of invasive cells in per frame was counted and analyzed. $\mathrm{n}=3$ for each group. Scale bar $=50 \mu \mathrm{m}$. Wound-healing assays were performed to determine the migration ability of (C) U251MG, and (D) U-87MG ATCC cells following treatment with 0 and $10 \mu \mathrm{M}$ apatinib. The migration rate was analyzed by Image $\mathrm{G}$ software. $\mathrm{n}=3$ for each group. Scale bar $=100 \mu \mathrm{m}$. ${ }^{* *} \mathrm{P}<0.01, \mathrm{vs}$. $0 \mu \mathrm{M}$ apatinib.

Collectively, these results indicate that apatinib significantly enhanced the antitumor activity of TMZ in glioma cancer cells.

\section{Discussion}

Seeing as glioma is hypervascular in nature, sunitinib and sorafenib have been used to control abnormal vasculature in clinical trials (https://clinicaltrials.gov) (10). However, internal resistance restricts the use of first-generation inhibitors of the VEGF-VEGFR pathway (19). Previous clinical studies have indicated the efficiency of apatinib treatment in recurrent glioma and that apatinib can transcend the brain-blood barrier $(11,20)$. However, its mechanism of action remained unclear. In the present study, antitumor activity was observed in apatinib-treated glioma cells (significant inhibition of cell proliferation and invasion). Apatinib was also demonstrated to improve the antitumor activity of TMZ in glioma cells. All experiments were performed using two glioma cell lines, which differ in mutation status. Collectively, the present study suggests that apatinib may provide a novel therapeutic strategy for glioma, particularly in combination with TMZ.

VEGFR2 is expressed in endothelial cells and upregulated in tumor vasculature (21). Phosphorylation of VEGFR2 by autocrine or paracrine VEGF has been demonstrated to activate the ERK, Akt, FAK, and MAPK pathways, which regulate cell proliferation and migration (21). Seeing as VEGFR2 is highly expressed in glioma cells (22), it is hypothesized that it serves a critical role in invasion and proliferation. The VEGFR2 pathway tyrosine kinase inhibitors (TKIs), sunitinib and sorafenib, have been previously used to treat patients with glioma (11). In the present study, it was demonstrated that VEGFR2 is highly expressed in glioma cells, and apatinib was revealed to inhibit p-VEGFR2, p-Akt and p-ERK protein expression in $\mathrm{U} 251 \mathrm{MG}$ and $\mathrm{U}-87 \mathrm{MG}$ ATCC cells in a concentration-dependent manner. Apatinib also significantly inhibited glioma cell proliferation and colony formation, and induced cell apoptosis in p53- and EGFR-mutated U251MG 
A
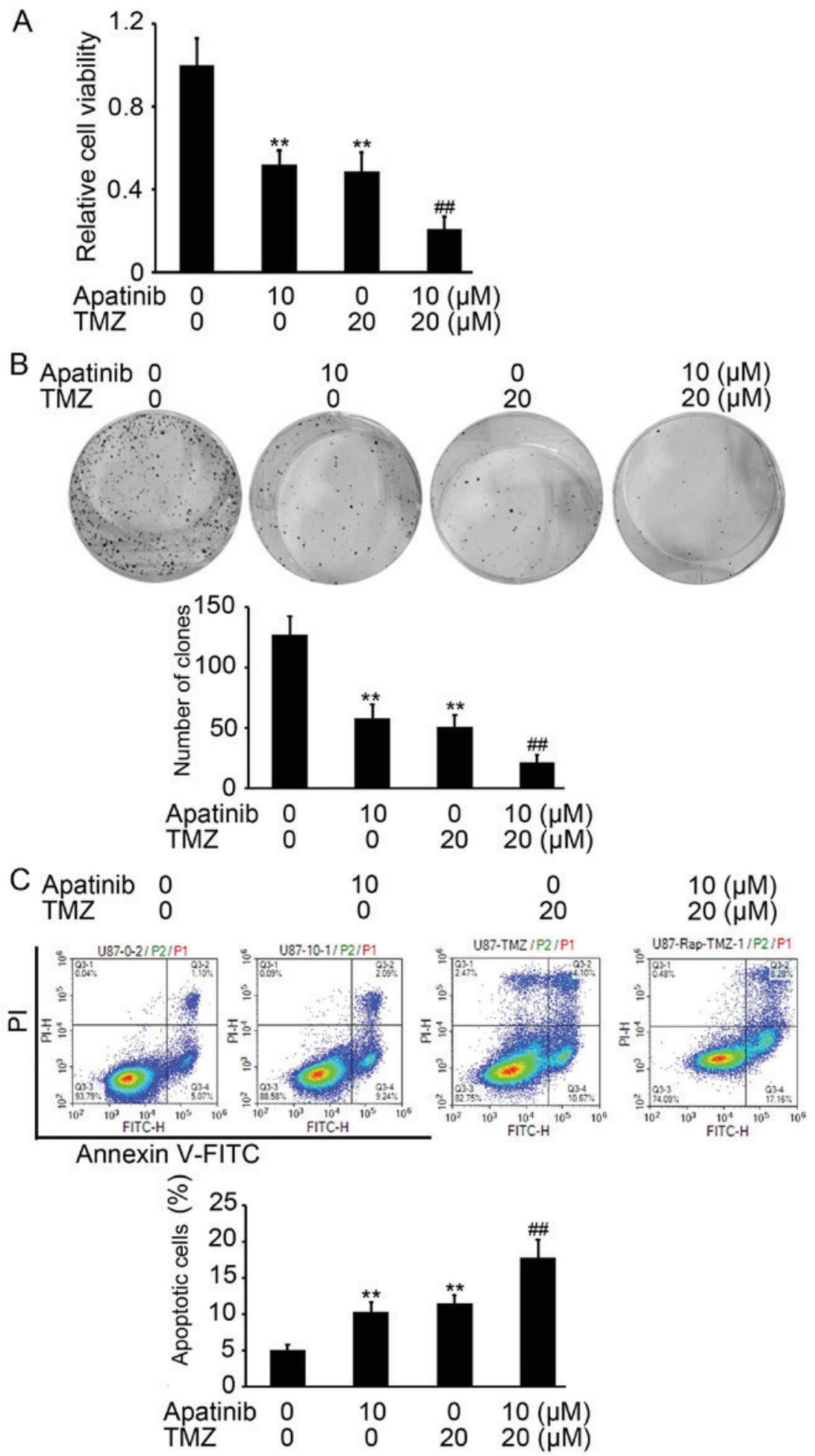

Figure 4. Apatinib enhances TMZ-mediated cell growth inhibition. (A) Viability of U-87MG ATCC cells was measured following treatment with 0 and $10 \mu \mathrm{M}$ apatinib and 0 and $20 \mu \mathrm{M}$ TMZ for $48 \mathrm{~h}$, relative to untreated cells. $\mathrm{n}=3$ for each group. (B) Colony formation assays were performed on U-87MG ATCC cells treated with 0 and $10 \mu \mathrm{M}$ apatinib and 0 and $20 \mu \mathrm{M}$ TMZ. The number of colonies was counted and analyzed. $\mathrm{n}=3$ for each group. (C) The proportion of apoptotic cells, analyzed by Annexin-V/PI staining in U-87MG ATCC cells treated with 0 and $10 \mu \mathrm{M}$ apatinib and 0 and $20 \mu \mathrm{M} \mathrm{TMZ}$. n=3 for each group. ${ }^{* *} \mathrm{P}<0.01$, vs. untreated group. ${ }^{\# \#} \mathrm{P}<0.01$, vs. TMZ-treated group. TMZ, temozolomide; PI, proprium iodide.

and wild-type U-87MG ATCC cells. Furthermore, inhibition of glioma-cell invasion and migration of glioma cells was observed in apatinib-treated glioma cells. These results provide evidence of the antitumor activity of apatinib in glioma cells. However, further in vivo studies are required to confirm this antitumor activity of apatinib.
Acquired resistance to temozolomide remains a limitation in the treatment of glioblastoma; approximately $90 \%$ recurrent glioblastoma cases are resistant to additional TMZ therapy (23). Type-selective endothelin receptor antagonists combined with chemotherapy have been investigated as a therapeutic strategy to improve clinical outcomes for 
A

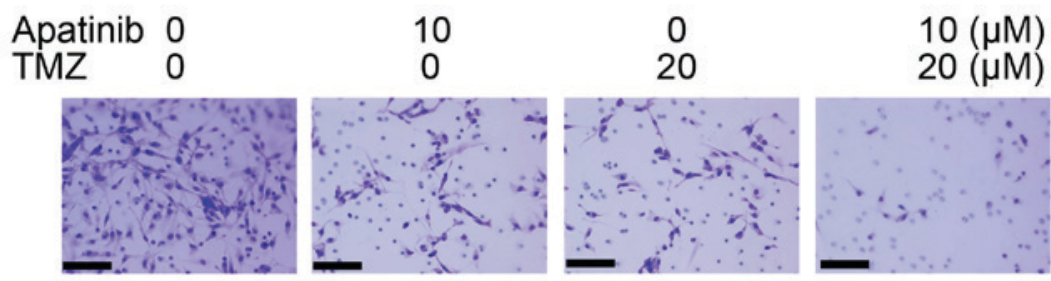

B

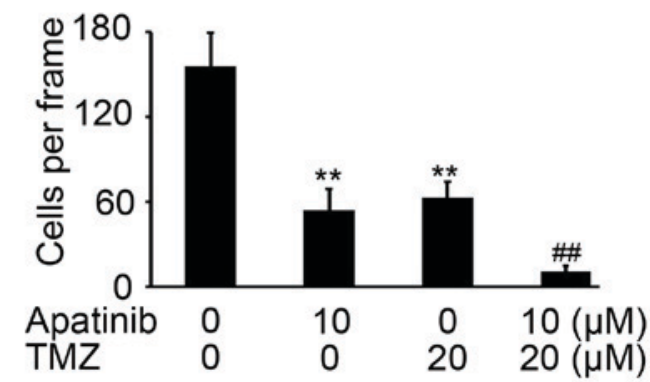

Figure 5. Apatinib enhances TMZ-mediated inhibition of cell invasion. (A) Matrigel-based invasion assays were performed to determine the invasive ability of U-87MG ATCC cells treated with 0 and $10 \mu \mathrm{M}$ apatinib and 0 and $20 \mu \mathrm{M}$ TMZ. Scale bar, $50 \mu \mathrm{m}$. (B) The number of invasive cells per frame was counted and analyzed. $\mathrm{n}=3$ for each group. ${ }^{* *} \mathrm{P}<0.01$, vs. untreated cells; ${ }^{\# \#} \mathrm{P}<0.01$, vs. TMZ-treated cells. TMZ, temozolomide.

patients with glioma (24). Macitentan, a dual endothelin receptor antagonist, combined with temozolomide treatment is well-tolerated, produces a durable response, and warrants clinical evaluation in glioblastoma (25). Treatment of glioblastoma cells with ER stress-inducing drugs has been demonstrated to induce drug-sensitization of glioma cells to TMZ through downregulation of O-6-methylguanine-DNA methyltransferase, N-methylpurine DNA glycosylase and Rad51 recombinase (26). The present study suggests that the addition of apatinib to TMZ may enhance the effect of chemotherapy. Treatment of glioma cells with apatinib or TMZ alone resulted increased apoptosis and decreased proliferation and invasion compared with untreated cells. Notably, the combination of apatinib and TMZ resulted in enhanced inhibition of cell proliferation and invasion compared with TMZ treatment alone. These findings demonstrated a synergistic antitumor effect of apatinib and TMZ in glioma cells and may explain the clinical benefits observed following combination treatment of recurrent glioma (24). Further in vivo studies are required to demonstrate the synergistic antitumor effect of apatinib and TMZ in glioma.

Previous studies have demonstrated enhanced activation of Akt and ERK in TMZ-resistant glioma cells $(27,28)$. Overexpression of Akt suppressed the enhanced cytotoxic effect of TMZ mediated by SRC-silencing (29). Treatment with the estrogen receptor- $\beta$ agonist (liquiritigenin) was demonstrated to enhance TMZ-sensitivity of glioma cells by inhibiting PI3K/Akt/mTOR pathway (30). TGF- $\beta 1$-dependent activation of SMAD/ERK signaling is involved in connective tissue growth factor-mediated TMZ-resistance in glioma (31). Targeting of the MEK-ERK-MDM2-p53 signaling pathway in combination with TMZ has been suggested to be a novel and promising therapeutic strategy in the treatment of glioblastoma (32). In the present study, inhibition of Akt/ERK activation by apatinib was demonstrated in glioma cells, which may contribute to the synergistic antitumor effect of apatinib and TMZ in glioma.
In summary, apatinib demonstrated efficient antitumor activity and enhanced the effect of TMZ in glioma cells, which was associated with decreased cell proliferation, colony formation, invasion and migration, and increased cell apoptosis. This antitumor activity of apatinib was observed in p53- and EGFR-mutated cells, as well as wild-type cells. These results provide a basis for the clinical use of apatinib in glioma treatment. Further in vivo studies are required to characterize the clinical antitumor activity of apatinib in glioma.

\section{Acknowledgements}

Not applicable.

\section{Funding}

The present study was supported by the National Natural Science Foundation of China (grant no. 81602186) and the China Postdoctoral Science Foundation (grant no. 2017M612216).

\section{Availability of data and materials}

All of the datasets generated/analyzed in the present study are included in the published manuscript.

\section{Authors' contributions}

$\mathrm{CW}, \mathrm{MJ}, \mathrm{XZ}, \mathrm{HH}, \mathrm{QL}$ and ZY contributed to data acquisition, the analysis and interpretation of data, were involved in drafting and writing the manuscript, revising it critically for important intellectual content and gave the final approval of the version to be published. ZY and XZ were accountable for design of this study and all aspects of the work in ensuring that questions related to the accuracy or integrity of any part of the work are appropriately investigated and resolved. 


\section{Ethics approval and consent to participate}

Not applicable.

\section{Consent for publication}

Not applicable.

\section{Competing interests}

The authors declare that they have no competing interests.

\section{References}

1. Huang Y, Rajappa P, Hu W, Hoffman C, Cisse B, Kim JH, Gorge E, Yanowitch R, Cope W, Vartanian E, et al: A proangiogenic signaling axis in myeloid cells promotes malignant progression of glioma. J Clin Invest 127: 1826-1838, 2017.

2. Wick W, Weller M, van den Bent M, Sanson M, Weiler M, von Deimling A, Plass C, Hegi M, Platten M and Reifenberger G: MGMT testing-the challenges for biomarker-based glioma treatment. Nat Rev Neurol 10: 372-385, 2014.

3. Watkins S and Sontheimer H: Unique biology of gliomas: Challenges and opportunities. Trends Neurosci 35: 546-556, 2012.

4. Jiang T, Mao Y, Ma W, Mao Q, You Y, Yang X, Jiang C, Kang C, Li X, Chen L, et al: CGCG clinical practice guidelines for the management of adult diffuse gliomas. Cancer Lett 375: 263-273, 2016.

5. Skalsky RLand Cullen BR: Reduced expression of brain-enriched microRNAs in glioblastomas permits targeted regulation of a cell death gene. PLoS One 6: e24248, 2011.

6. Ji Y, Vogel RI and Lou E: Temozolomide treatment of pituitary carcinomas and atypical adenomas: Systematic review of case reports. Neurooncol Pract 3: 188-195, 2016.

7. Newlands ES, Stevens MF, Wedge SR, Wheelhouse RT and Brock C: Temozolomide: A review of its discovery, chemical properties, pre-clinical development and clinical trials. Cancer Treat Rev 23: 35-61, 1997.

8. Chan JA, Stuart K, Earle CC, Clark JW, Bhargava P, Miksad R, Blaszkowsky L, Enzinger PC, Meyerhardt JA, Zheng H, et al Prospective study of bevacizumab plus temozolomide in patients with advanced neuroendocrine tumors. J Clin Oncol 30: 2963-2968, 2012.

9. Gilbert MR, Wang M, Aldape KD, Stupp R, Hegi ME, Jaeckle KA, Armstrong TS, Wefel JS, Won M, Blumenthal DT, et al: Dose-dense temozolomide for newly diagnosed glioblastoma: A randomized phase III clinical trial. J Clin Oncol 31: 4085-4091, 2013.

10. Khasraw M, Simeonovic M and Grommes C: Bevacizumab for the treatment of high-grade glioma. Expert Opin Biol Ther 12: 1101-1111, 2012

11. Chen JH, Yu GF, Jin SY, Zhang WH, Lei DX, Zhou SL and Song XR: Activation of $\alpha 2$ adrenoceptor attenuates lipopolysaccharide-induced hepatic injury. Int J Clin Exp Pathol 8: 10752-10759, 2015.

12. Peng H, Zhang Q, Li J, Zhang N, Hua Y, Xu L, Deng Y, Lai J, Peng Z, Peng B, et al: Apatinib inhibits VEGF signaling and promotes apoptosis in intrahepatic cholangiocarcinoma. Oncotarget 7: 17220-17229, 2016.

13. Kim KL and Suh W: Apatinib, an inhibitor of vascular endothelial growth factor receptor 2, suppresses pathologic ocular neovascularization in mice. Invest Ophthalmol Vis Sci 58: 3592-3599, 2017

14. Zhou N, Liu C, Hou H, Zhang C, Liu D, Wang G, Liu K, Zhu J, Lv H, Li T and Zhang X: Response to apatinib in chemotherapy-failed advanced spindle cell breast carcinoma. Oncotarget 7: 72373-72379, 2016.

15. Huang L, Wei Y, Shen S, Shi Q, Bai J, Li J, Qin S, Yu H and Chen F: Therapeutic effect of apatinib on overall survival is mediated by prolonged progression-free survival in advanced gastric cancer patients. Oncotarget 8: 29346-29354, 2017.
16. Allen M, Bjerke M, Edlund H, Nelander S and Westermark B: Origin of the U87MG glioma cell line: Good news and bad news. Sci Transl Med 8: 354re353, 2016.

17. Nitta Y, Shimizu S, Shishido-Hara Y, Suzuki K, Shiokawa Y and Nagane M: Nimotuzumab enhances temozolomide-induced growth suppression of glioma cells expressing mutant EGFR in vivo. Cancer Med 5: 486-499, 2016.

18. Dai L, Cui X, Zhang X, Cheng L, Liu Y, Yang Y, Fan P, Wang Q, Lin Y, Zhang J, et al: SARI inhibits angiogenesis and tumour growth of human colon cancer through directly targeting ceruloplasmin. Nat Commun 7: 11996, 2016.

19. Dietrich J, Norden AD and Wen PY: Emerging antiangiogenic treatments for gliomas-efficacy and safety issues. Curr Opin Neurol 21: 736-744, 2008

20. Hu X, Zhang J, Xu B, Jiang Z, Ragaz J, Tong Z, Zhang Q, Wang X, Feng J, Pang D, et al: Multicenter phase II study of apatinib, a novel VEGFR inhibitor in heavily pretreated patients with metastatic triple-negative breast cancer. Int J Cancer 135: 1961-1969, 2014

21. Fontanella C, Ongaro E, Bolzonello S, Guardascione M, Fasola G and Aprile G: Clinical advances in the development of novel VEGFR2 inhibitors. Ann Transl Med 2: 123, 2014.

22. Shankar A, Jain M, Lim MJ, Angara K, Zeng P, Arbab SA, Iskander A, Ara R, Arbab AS and Achyut BR: Anti-VEGFR2 driven nuclear translocation of VEGFR2 and acquired malignant hallmarks are mutation dependent in glioblastoma. J Cancer Sci Ther 8: 172-178, 2016

23. Wen PY and Kesari S: Malignant gliomas in adults. N Engl J Med 359: 492-507, 2008.

24. Alshami J, Guiot MC, Owen S, Kavan P, Gibson N, Solca F, Cseh A, Reardon DA and Muanza T: Afatinib, an irreversible ErbB family blocker, with protracted temozolomide in recurrent glioblastoma: A case report. Oncotarget 6: 34030-34037, 2015.

25. Kim SJ, Lee HJ, Kim MS, Choi HJ, He J, Wu Q, Aldape K, Weinberg JS, Yung WK, Conrad CA, et al: Macitentan, a dual endothelin receptor antagonist, in combination with temozolomide leads to glioblastoma regression and long-term survival in mice. Clin Cancer Res 21: 4630-4641, 2015.

26. Xipell E, Aragón T, Martinez-Velez N, Vera B, Idoate MA, Martinez-Irujo JJ, Garzón AG, Gonzalez-Huarriz M, Acanda AM, Jones C, et al: Endoplasmic reticulum stress-inducing drugs sensitize glioma cells to temozolomide through downregulation of MGMT, MPG, and Rad51. Neuro Oncol 18: 1109-1119, 2016.

27. Han S, Li Z, Master LM, Master ZW and Wu A: Exogenous IGFBP-2 promotes proliferation, invasion, and chemoresistance to temozolomide in glioma cells via the integrin $\beta 1$-ERK pathway. Br J Cancer 111: 1400-1409, 2014.

28. Vo VA, Lee JW, Lee HJ, Chun W, Lim SY and Kim SS: Inhibition of JNK potentiates temozolomide-induced cytotoxicity in U87MG glioblastoma cells via suppression of Akt phosphorylation. Anticancer Res 34: 5509-5515, 2014.

29. Wang Z, Sun J, Li X, Yang S, Yue S, Zhang J, Yang X, Zhu T, Jiang $R$ and Yang W: Downregulation of Src enhances the cytotoxic effect of temozolomide through AKT in glioma. Oncol Rep 29: 1395-1398, 2013.

30. Liu X, Wang L, Chen J, Ling Q, Wang H, Li S, Li L, Yang S, Xia M and Jing L: Estrogen receptor $\beta$ agonist enhances temozolomide sensitivity of glioma cells by inhibiting PI3K/AKT/mTOR pathway. Mol Med Rep 11: 1516-1522, 2015.

31. Zeng H, Yang Z, Xu N, Liu B, Fu Z, Lian C and Guo H: Connective tissue growth factor promotes temozolomide resistance in glioblastoma through TGF- $\beta 1$-dependent activation of Smad/ERK signaling. Cell Death Dis 8: e2885, 2017.

32. Sato A, Sunayama J, Matsuda K, Seino S, Suzuki K, Watanabe E, Tachibana K, Tomiyama A, Kayama T and Kitanaka C: MEK-ERK signaling dictates DNA-repair gene MGMT expression and temozolomide resistance of stem-like glioblastoma cells via the MDM2-p53 axis. Stem Cells 29: 1942-1951, 2011.

This work is licensed under a Creative Commons Attribution-NonCommercial-NoDerivatives 4.0 International (CC BY-NC-ND 4.0) License. 